\title{
The Role of Natural Killer Cells in Inflammation in Active Adult-onset Still Disease
}
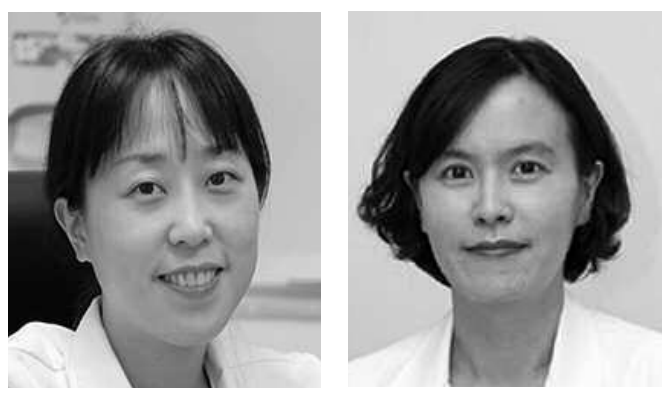

Adult-onset Still disease (AOSD) is a systemic inflammatory disease characterized by spiking fever, arthralgia, rash, leukocytosis, and hepatosplenomegaly, similar to systemic-onset juvenile idiopathic arthritis (sJIA) ${ }^{1,2}$. The etiology of AOSD is unclear but the disease is thought to result from a combination of genetic and environmental factors. The disease pathology is characterized by widespread immune dysregulation, involving cytokines and their receptors, immune cells, and tissues. Activation of the innate immune system plays a major role in the systemic inflammation that characterizes AOSD and sJIA ${ }^{3}$. Activated macrophages and neutrophils induce increased levels of proinflammatory cytokines, such as interleukin (IL)-1 $\beta$, IL-18, IL-6, and interferon (IFN)- $\gamma$. These proinflammatory cytokines provoke activation of macrophages and neutrophils, and differentiation of $\mathrm{T}$ cells. Decreased numbers and functional impairment of natural killer (NK) cells have also been implicated in the pathogenesis of AOSD and sJIA ${ }^{4,5}$.

NK cells are an important lymphocytic component of the innate immune system, providing a cytotoxicity defense against malignant, infected, and autoreactive cells ${ }^{6,7}$. NK cells exert their effects by secreting cytolytic granules such as perforin, granzyme, and serine proteases, and induce caspase-dependent apoptosis through death receptors including Fas/FasL. Impaired cytotoxic function of NK cells leads to excessive activation of macrophages and T cells, as seen in macrophage activation syndrome (MAS), and is a major, life-threatening complication of AOSD and sJIA ${ }^{8,9}$. Primary hemophagocytic lymphohistiocytosis (HLH), a hereditary disorder of MAS, occurs as a result of genetic defects in the perforin- or granzyme-dependent pathways of NK cells or cytotoxic T cells ${ }^{10}$. Additional genetic mutations associated with inhibitory or stimulatory NK cell receptors have also been observed in secondary HLH. The resulting failure of NK cells to lyse activated antigen-presenting cells results in sustained stimulation of lymphocytes and macrophages, leading to a cascade of inflammatory cytokines. Taken together, these data suggest that the dysfunction of NK cells or NK T cells may be important in the underlying systemic inflammation and macrophage activation seen in chronic inflammatory disorders, such as sJIA and AOSD. Alteration of NK cells has been well documented in AOSD and sJIA ${ }^{11}$, with active AOSD patients exhibiting decreased circulating NK T cells along with NK cells with poor proliferative function and impaired cytotoxicity ${ }^{4,5}$. Such defective NK cytotoxicity was confirmed by the impaired IL-18-induced IFN- $\gamma$ production seen in patients with $\mathrm{sJIA}^{12}$. Further, downregulated NK T or NK cell populations recovered during remission of SJIA or $\mathrm{AOSD}^{4,13}$.

In this issue of The Journal, Shimojima, et al investigated the characteristics of circulating NK cells, including IFN- $\gamma$ production, in patients with active $\mathrm{AOSD}^{14}$. Based on flow cytometry data, Shimojima, et al showed that the number of IFN- $\gamma$ expressing NK cells was increased in acute AOSD compared to healthy controls (HC), although the proportion of circulating NK cells as a percentage of total lymphocytes was decreased. Further, expression of IL-12 and IL-15 receptors, but not of IL-18 receptors, was significantly increased on NK cells in acute AOSD. The cytotoxicity of NK cells depends on IFN- $\gamma$, with IL-18, IL-12, and IL-15 modulating the secretion of IFN- $\gamma$ by interacting with their receptors on NK cells ${ }^{15}$. A defect in the phosphorylation of IL-18 receptor- $\beta$ on NK cells has been reported in sJIA ${ }^{16}$, and may contribute to impaired NK cell expansion in AOSD despite the high levels of IL-18 seen in these patients. Similarly, that study also found that increased IL-18 levels were not correlated with the number of IFN- $\gamma$-expressing NK cells. The accompanying increases in IL-12 and IL-15 receptor expression on NK cells could be regarded as a compensatory reaction against high levels of intracellular IFN- $\gamma$. These data show that the persistent upregulation of IL-18 in acute AOSD does not affect NK cell expression levels, despite the higher numbers of IFN- $\gamma$-expressing NK cells in these patients.

NK cells are divided into 5 phenotypes based on differences in surface receptor expression and cytotoxic

See NK cells in AOSD, page 1268

Personal non-commercial use only. The Journal of Rheumatology Copyright @ 2019 . All rights reserved. 
functions ${ }^{17}$. CD56 ${ }^{\mathrm{dim}}$ cells exhibit more potent cytolytic activity and contain more cytoplasmic granules than CD56 $6^{\text {bright }}$ cells, which have higher proliferative potential and show enhanced cytokine production ${ }^{18}$. Shimojima, et al divided NK cells into 2 distinct subsets according to CD56 expression, and enhanced their IFN- $\gamma$-producing ability ${ }^{14}$. IFN- $\gamma$ expression in CD56 ${ }^{\text {bright }}$ cells was significantly decreased in acute AOSD compared to HC, while IFN- $\gamma$ expression in CD56 ${ }^{\text {dim }}$ was significantly increased. This result suggested 2 basic hypotheses: that CD56 ${ }^{\mathrm{dim}} \mathrm{NK}$ cells develop their ability to produce IFN- $\gamma$ during AOSD development in response to other immunopathogenic cells, and that the absolute number of NK cells expressing IFN- $\gamma$ can be altered during the transition from CD56 $6^{\text {bright }}$ to CD56 ${ }^{\text {dim }}$ in acute AOSD. While the lower proportion of CD56 bright cells could be regarded as a paradoxical phenomenon due to their higher IFN- $\gamma$ expression, this effect may be due to a lack of immunomodulating cytokines such as IL-10 and IL-13, which are induced by CD56 $6^{\text {bright }}$ cells ${ }^{19}$. Further, the lack of regulatory CD56 $6^{\text {bright }} \mathrm{NK}$ cells might exacerbate the inflammatory response in AOSD. To confirm this hypothesis, in vitro experiments should be conducted under various conditions.

The data from Shimojima, et al revealed some limitations regarding the extent of the role of NK cells in the pathogenesis of AOSD. First, they evaluated the total number of NK cells and IFN- $\gamma$-expressing NK cells, along with IL-12, IL-15, and IL-18 receptor expression levels in each NK cell population; however, functional studies of each NK cell population were not performed. NK cells are a critical cytotoxic regulator of the immune response, with NK cell dysfunction thought to be an important factor in AOSD. A better understanding of differences in cytotoxic function based on IFN- $\gamma$ production in NK cells, and of NK cell frequency, may be important for evaluating the role of these cells in AOSD pathogenesis. Second, the study evaluated only the association between NK cell characteristics and acute inflammation in AOSD. Recently, AOSD was shown to exhibit 2 distinct patterns of disease progression: systemic inflammatory and chronic articular patterns ${ }^{2}$. The systemic inflammatory pattern is characterized by recurrent systemic flares, such as fever, rash, and myalgia, while the chronic articular pattern is characterized by persistent symptoms of erosive arthritis, similar to rheumatoid arthritis ${ }^{3}$. Many researchers have suggested that NK cell dysfunction could be related to systemic inflammation, rather than the chronic articular pattern of AOSD pathogenesis ${ }^{2}$. However, in the cited study ${ }^{14}$, patients experiencing AOSD flares during followup were not included, and the patterns of AOSD were not described. Third, Shimojima, et al showed that circulating NK cell counts were negatively correlated with AOSD activity when comparing active and remission status; however, the authors did not consider the influence of treatment, particularly with glucocorticoids. Glucocorticoids were shown to suppress initial release of IFN- $\gamma$ from NK cells, and to enhance cell proliferation and survival in the presence of IL-2 and IL-12 ${ }^{20}$. Considering that glucocorticoids are the treatment of choice in AOSD, changes in the population or function of NK cells might be affected by administration of these agents. Intriguingly, the levels of IFN- $\gamma$-producing NK cells were decreased after remission in almost all patients with AOSD, with 4 of 7 patients in remission currently receiving glucocorticoids as a maintenance therapy. Therefore, to rule out an effect of glucocorticoids, greater numbers of followup patients in remission not treated with corticosteroids should be recruited, to confirm the number of IFN- $\gamma$-producing NK cells in AOSD remission ${ }^{14}$. Although Shimojima, et al suggested a possible connection between changes in the levels of cytokines (such as IL-18) and NK cell characteristics, they failed to demonstrate a clear linkage mechanism because of the absence of in vitro functional studies. Finally, the number of subjects in their study was small, and peripheral blood cells in patients with remission status could be affected by a variety of factors, such as the types of drugs administered.

Several analyses revealed impaired NK cell cytotoxicity and decreased NK cell number in acute AOSD (Table 1); however, the cause of impaired NK cell cytotoxicity and decreased NK cell numbers remains unclear. Evidence regarding the underlying cause of such changes in NK cells, both in patients with active AOSD and those in remission, and of the associations between cytokine levels and NK cell cytotoxicity in AOSD and sJIA, is sorely lacking. Possible causes of these phenomena include pathologic alterations,

Table 1. Current findings regarding the role of natural killer cells in adultonset Still disease (AOSD) and systemic juvenile idiopathic arthritis (sJIA).

\begin{tabular}{|c|c|c|}
\hline Disease & Findings & Ref \\
\hline \multirow[t]{2}{*}{ AOSD } & Lower NK T cell counts & 4 \\
\hline & Defect in GalCer-mediated NK cytotoxicity & \\
\hline \multirow[t]{2}{*}{ AOSD } & Lower NK cell counts & 5 \\
\hline & Lower NK cytolytic function & \\
\hline \multirow[t]{2}{*}{ sJIA } & Lower NK cytolytic activity & 9 \\
\hline & Lower circulating CD56 ${ }^{\text {bright }} \mathrm{NK}$ cell levels & \\
\hline \multirow[t]{2}{*}{ sJIA } & $\begin{array}{l}\text { Impaired upregulation of cell-mediated perforin } \\
\text { and IFN- } \gamma \text { in NK cells }\end{array}$ & 16 \\
\hline & Defect in IL-18 receptor $\beta$ phosphorylation & \\
\hline \multirow[t]{2}{*}{ sJIA } & $\begin{array}{l}\text { Lower cell counts, cytotoxicity, perforin, and } \\
\text { granzyme B expression in NK cells }\end{array}$ & 13 \\
\hline & Lower frequency of KIR2DS4 & \\
\hline \multirow[t]{5}{*}{ sJIA } & Increased expression of innate genes and decreased & \\
\hline & expression of immune-regulating genes of NK cells & 12 \\
\hline & Alterations in inhibitory and excitatory receptors of & \\
\hline & Decreased granzyme K expression in CD56 $6^{\text {bright }}$ & \\
\hline & NK cells and defective IL- 18 -induced IFN- $\gamma$ production & \\
\hline sJIA & Impaired NK cell activation by IL-18 & 21 \\
\hline
\end{tabular}

NK: natural killer; IL: interleukin; KIR: killer cell immunoglobulin-like receptor; IFN: interferon; KIR2DS4: killer cell immunoglobulin-like excitatory receptors.

Personal non-commercial use only. The Journal of Rheumatology Copyright @ 2019. All rights reserved. 
including the release of lower levels of IFN- $\gamma$ from NK cells, as well as effects of genetic susceptibilities on NK cell function; however, data on the effects of genetic factors on NK cell cytotoxicity in SJIA and AOSD remain scarce.

The NK cell machinery includes a variety of receptors with both inhibitory and excitatory actions, which together control NK cell activation. A study examining gene expression in patients with AOSD identified a specific gene polymorphism in NK cell receptors ${ }^{13}$. Similarly, an in vitro assay examining killer cell immunoglobulin-like receptors (KIR), which are composed of inhibitory and excitatory receptors, revealed lower levels of the excitatory receptor KIR2DS4 in sJIA compared to polyarticular and pauciarticular JIA. While such genetic differences may lead to uncontrolled innate immune activation, more conclusive evidence regarding the genetic susceptibility of NK cells in AOSD is required.

A better understanding of the role of NK cells in the pathogenesis of AOSD is necessary for proper diagnosis and treatment of patients with AOSD. Despite certain limitations in the data, this study has improved our understanding of NK cell characteristics and IFN- $\gamma$ production patterns in AOSD $^{14}$. The data presented in this article help to clarify the details regarding IFN- $\gamma$ production according to the expression of various receptors and NK cell subsets in AOSD. However, it remains unclear whether the observed changes in NK cells are associated with disease etiology or arise as a result of the chronic immune dysregulation seen in this disease. Ongoing genetic and molecular investigations into these various aspects of AOSD pathology may help to explain the changes in NK cells seen therein, and their roles in disease pathogenesis.

\section{JU-YANG JUNG ${ }^{(i), ~ M D, ~ P h D, ~}$ \\ Assistant Professor, \\ Department of Rheumatology, \\ Ajou University Medical School;}

HYOUN-AH KIM ${ }^{10}$, MD, $\mathrm{PhD}$,

Associate Professor,

Department of Rheumatology,

Ajou University Medical School,

Suwon, Korea.

This work was supported by grants from the Basic Science Research Program through the National Research Foundation of Korea, funded by the Ministry of Education, Science and Technology (grant number 2019R1A2C1005988). Address correspondence to Dr. H.A. Kim, Department of Rheumatology, Ajou University School of Medicine, 164 Worldcup-ro, Yeongtong-gu, Suwon 16499, Korea.

E-mail: nakhada@naver.com

\section{REFERENCES}

1. Giacomelli R, Ruscitti P, Shoenfeld Y. A comprehensive review on adult onset Still's disease. J Autoimmun 2018;93:24-36.

2. Gerfaud-Valentin M, Jamilloux Y, Iwaz J, Seve P. Adult-onset Still's disease. Autoimmun Rev 2014;13:708-22.

3. Ruscitti P, Giacomelli R. Pathogenesis of adult onset Still's disease: current understanding and new insights. Expert Rev Clin Immunol 2018;14:965-76.

4. Lee SJ, Cho YN, Kim TJ, Park SC, Park DJ, Jin HM, et al. Natural killer T cell deficiency in active adult-onset Still's Disease: correlation of deficiency of natural killer T cells with dysfunction of natural killer cells. Arthritis Rheum 2012;64:2868-77.

5. Park JH, Kim HS, Lee JS, Kim JJ, Jung KH, Park YW, et al. Natural killer cell cytolytic function in Korean patients with adult-onset Still's disease. J Rheumatol 2012;39:2000-7.

6. Popko K, Gorska E. The role of natural killer cells in pathogenesis of autoimmune diseases. Cent Eur J Immunol 2015;40:470-6.

7. Caligiuri MA. Human natural killer cells. Blood 2008;112:461-9.

8. Cifaldi L, Prencipe G, Caiello I, Bracaglia C, Locatelli F, De Benedetti $\mathrm{F}$, et al. Inhibition of natural killer cell cytotoxicity by interleukin-6: implications for the pathogenesis of macrophage activation syndrome. Arthritis Rheumatol 2015;67:3037-46.

9. Villanueva J, Lee S, Giannini EH, Graham TB, Passo MH, Filipovich A, et al. Natural killer cell dysfunction is a distinguishing feature of systemic onset juvenile rheumatoid arthritis and macrophage activation syndrome. Arthritis Res 2005;7:R30-7.

10. Filipovich AH, Chandrakasan S. Pathogenesis of hemophagocytic lymphohistiocytosis. Hematol Oncol Clin North Am 2015; 29:895-902.

11. Avau A, Put K, Wouters CH, Matthys P. Cytokine balance and cytokine-driven natural killer cell dysfunction in systemic juvenile idiopathic arthritis. Cytokine Growth Factor Rev 2015;26:35-45.

12. Put K, Vandenhaute J, Avau A, van Nieuwenhuijze A, Brisse E, Dierckx T, et al. Inflammatory gene expression profile and defective interferon-gamma and granzyme $\mathrm{K}$ in natural killer cells from systemic juvenile idiopathic arthritis patients. Arthritis Rheumatol 2017;69:213-24.

13. Zhou J, Tang X, Ding Y, An Y, Zhao X. Natural killer cell activity and frequency of killer cell immunoglobulin-like receptors in children with different forms of juvenile idiopathic arthritis. Pediatr Allergy Immunol 2013;24:691-6.

14. Shimojima Y, Kishida D, Ueno KI, Ushiyama S, Ichikawa T, Sekijima Y. Characteristics of circulating natural killer cells and their interferon- $\gamma$ production in active adult-onset Still disease. J Rheumatol 2019;46:1268-76.

15. Berrien-Elliott MM, Wagner JA, Fehniger TA. Human cytokine-induced memory-like natural killer cells. J Innate Immun 2015;7:563-71.

16. de Jager W, Vastert SJ, Beekman JM, Wulffraat NM, Kuis W, Coffer PJ, et al. Defective phosphorylation of interleukin-18 receptor beta causes impaired natural killer cell function in systemic-onset juvenile idiopathic arthritis. Arthritis Rheum 2009;60:2782-93.

17. Zhang C, Tian Z. NK cell subsets in autoimmune diseases. J Autoimmun 2017;83:22-30.

18. Moretta L. Dissecting CD56dim human NK cells. Blood 2010;116:3689-91.

19. Wilk E, Kalippke K, Buyny S, Schmidt RE, Jacobs R. New aspects of NK cell subset identification and inference of NK cells' regulatory capacity by assessing functional and genomic profiles. Immunobiol 2008;213:271-83.

20. Morgan DJ, Davis DM. Distinct effects of dexamethasone on human natural killer cell responses dependent on cytokines. Front Immunol 2017;8:432.

21. Takakura M, Shimizu M, Yakoyama T, Mizuta M, Yachie A. Transient natural killer cell dysfunction associated with interleukin-18 overproduction in systemic juvenile idiopathic arthritis. Pediatr Int 2018;60:984-5.

J Rheumatol 2019;46:1253-5; doi:10.3899/jrheum.190369 